\title{
On the Stability of Lung Parenchymal Lesions with Applications to Early Pneumothorax Diagnosis
}

\author{
Archis R. Bhandarkar, ${ }^{1}$ Rohan Banerjee, ${ }^{1}$ and Padmanabhan Seshaiyer ${ }^{2}$ \\ ${ }^{1}$ Thomas Jefferson High School for Science and Technology, Alexandria, VA 22312, USA \\ ${ }^{2}$ Mathematical Sciences, George Mason University, Fairfax, VA 22030, USA \\ Correspondence should be addressed to Archis R. Bhandarkar; archisrb@gmail.com
}

Received 1 December 2012; Revised 26 March 2013; Accepted 30 March 2013

Academic Editor: Thierry Busso

Copyright (c) 2013 Archis R. Bhandarkar et al. This is an open access article distributed under the Creative Commons Attribution License, which permits unrestricted use, distribution, and reproduction in any medium, provided the original work is properly cited.

\begin{abstract}
Spontaneous pneumothorax, a prevalent medical challenge in most trauma cases, is a form of sudden lung collapse closely associated with risk factors such as lung cancer and emphysema. Our work seeks to explore and quantify the currently unknown pathological factors underlying lesion rupture in pneumothorax through biomechanical modeling. We hypothesized that lesion instability is closely associated with elastodynamic strain of the pleural membrane from pulsatile air flow and collagen-elastin dynamics. Based on the principles of continuum mechanics and fluid-structure interaction, our proposed model coupled isotropic tissue deformation with pressure from pulsatile air motion and the pleural fluid. Next, we derived mathematical instability criteria for our ordinary differential equation system and then translated these mathematical instabilities to physically relevant structural instabilities via the incorporation of a finite energy limiter. The introduction of novel biomechanical descriptions for collagen-elastin dynamics allowed us to demonstrate that changes in the protein structure can lead to a transition from stable to unstable domains in the material parameter space for a general lesion. This result allowed us to create a novel streamlined algorithm for detecting material instabilities in transient lung CT scan data via analyzing deformations in a local tissue boundary.
\end{abstract}

\section{Introduction}

Spontaneous pneumothorax is a form of sudden lung collapse closely associated with risk factors such as lung cancer and emphysema. The disorder represents a pressing clinical challenge, affecting between 20 and $40 \%$ of patients with major trauma [1]. The onset of pneumothorax has been attributed to the rupture of quasi spherical parenchymal lesions or air blebs of the visceral pleural membrane [2]. The rupture of these lesions in turn releases air into the pleural cavity, and the consequent pressure buildup leads to the collapse of the lung [3]. Because pneumothorax has a relatively large recurrence rate of approximately $54 \%$ in the first four years after surgery, wholly understanding and quantifying the mechanisms behind lesion rupture are important [4].

The etiology and trigger mechanisms behind lesion rupture, however, still remain elusive $[2,5]$. We hypothesize that lesion rupture and instability are closely associated with elastodynamic strain of the visceral pleural membrane from pulsatile air flow and changes in the constitutive protein composition of tissue. In order to assess the validity of this hypothesis, we constructed a biomechanical model based on the principles of finite strain continuum mechanics and fluidstructure interaction. Our proposed model closely aligns with the behavior of true biological soft tissue through the coupling of membrane energy limitations and growth and remodeling driven by collagen-elastin dynamics. Through the use of an exhaustive ordinary differential equation stability analysis, we isolated several instability regions in the material parameter space of a general lesion.

Based on these mathematical results, we developed an algorithm to rapidly assay clinical lung CT scan data for structural instabilities. The algorithm is based on cataloging and processing local deformations in a tissue boundary isolated via Sobel Edge Detection. The local deformation of the boundary is then fit to our biomechanical model and registered as either stable or unstable based on whether or not the interpolated material parameters lie in the instability region. 


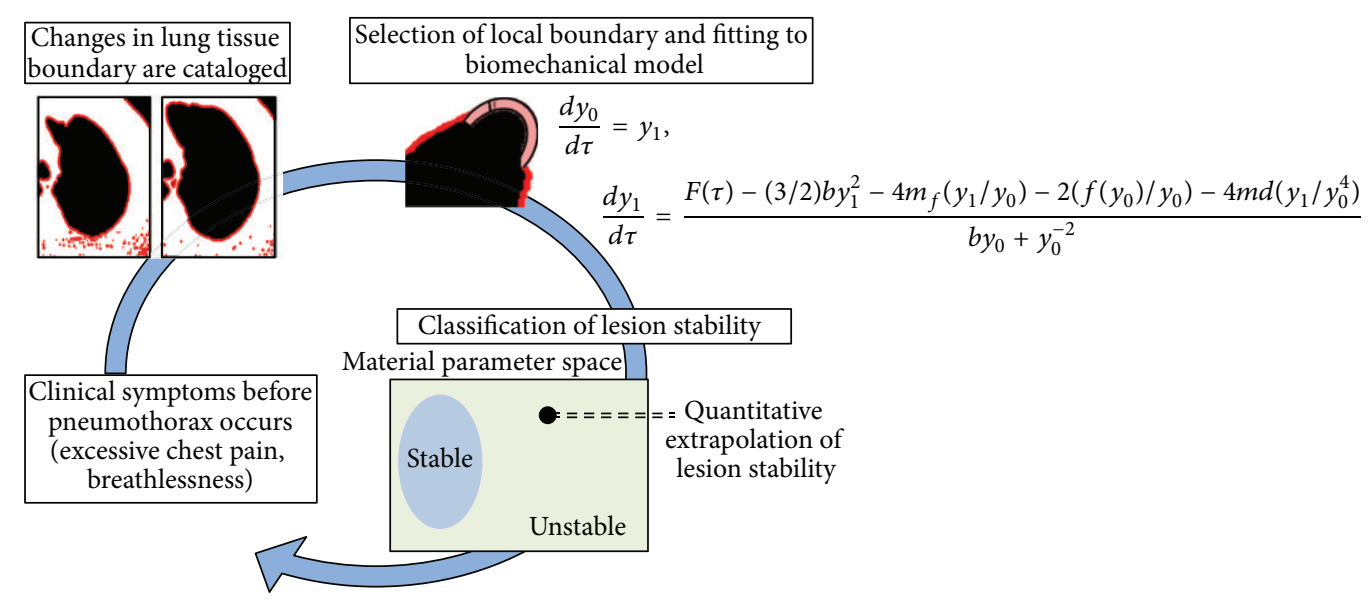

FIGURE 1: Outline of novel early diagnosis method. An outline of our novel diagnosis method. Based on the observation of certain symptoms, changes in the lung boundary are catalogued and material parameters are derived. The differential equation system is locally applied, and then a stability calculation is performed to determine the appropriate course of action.

Figure 1 shows a general outline of our novel early diagnostic method. In the future, the algorithm may be real time coupled with either ultrasound or CT scan data to quantify patient risk of pneumothorax earlier and improve the current diagnostic benchmark of only $75 \%$ lesion sensibility [6]. Unlike competitive diagnostic procedures for pneumothorax such as infrared thermography [1], our proposed method relies on detecting pathological hallmarks in tissue behavior before acute lung collapse actually occurs.

\section{Methodology}

Our study focused on the construction of a theoretically based biomechanical model for lung parenchymal lesion rupture and then the implementation of this model towards a streamlined algorithm for early pneumothorax diagnosis. We began with the theoretical development of a model for simple isotropic tissue deformation and then conducted several initial numerical investigations. Next, we derived mathematical instabilities for our system and then translated these mathematical instabilities to physically relevant structural instabilities via the incorporation of an energy limiter. We then incorporated collagen-elastin dynamics (dynamic alteration of the constitutive proteins in the tissue) to demonstrate how changes in tissue structure may lead to lesion rupture. Lastly, we developed an algorithm for determining lesion stability from CT scan data by fitting our biomechanical model onto tissue deformation data.

2.1. Mathematical Formulation. We modeled the parenchymal lesions with a spherical membrane geometry with thickness significantly smaller than the radius. Several parameters were needed in order to fully characterize the lesion geometry. The nondimensional stretch ratio $\lambda(t)$ was defined as $r(t) / R$, where $r(t)$ represents the deformed radius varying with time and $R$ is the original undeformed radius. Assuming membrane incompressibility, the constant volume condition
$V=4 \pi r^{2}(t) h(t)$ implies that the deformed thickness is $h(t)=H / \lambda(t)^{2}$, where $H$ represents the original undeformed thickness. Figure 2 depicts the geometry of these quasispherical lesions, including $r(t)$ and $h(t)$.

2.1.1. Constitutive Parenchymal Wall Model. Based on the findings of Tai and Lee [7], who concluded that lung tissue exhibits less than $10 \%$ anisotropy of the mean deformation, we pursued an isotropic model for the lung parenchymal wall. We had based a collagen-only isotropic pseudostrainenergy function for the wall of the parenchymal lesion on the work of Denny and Schroter [8]. Previous works by Denny and Schroter $[9,10]$ had also considered collagen and elastin using separate models [9], which were enhanced with viscoelastic contributions [10]. We adapted the DennySchroter constitutive relationship for our $1 \mathrm{D}$ formulation through multiplication by the deformed thickness $h$ :

$$
w=\frac{H}{\lambda(t)^{2}}\left(c_{1} \log \left[1-\left(\frac{e^{E_{11}}-1}{c_{2}}\right)\right]+c_{3} E_{11}\right)
$$

where $c_{1}$ and $c_{3}$ are constants with dimensions $\mathrm{N} \cdot \mathrm{m}^{-2}, c_{2}$ is dimensionless, and $E_{11}=\left(\lambda(t)^{2}-1\right) / 2$ (where $\mathbf{E}$ is the Green-Lagrangian strain tensor). Note that for simplicity, our model does not account for large stiffnesses for large strains. However, this is something that we hope to incorporate in future work. In order to facilitate the stability analysis and further calculations, this pseudostrain-energy function was approximated by a Taylor series centered about $E_{11}=0$, yielding

$$
\begin{aligned}
w= & \left(-\frac{c_{1} H}{c_{2}}+c_{3} H\right) E_{11}+\left(-\frac{c_{1} H}{2 c_{2}^{2}}+\frac{3 c_{1} H}{2 c_{2}}-2 c_{3} H\right) E_{11}^{2} \\
& +O\left[E_{11}^{3}\right] .
\end{aligned}
$$




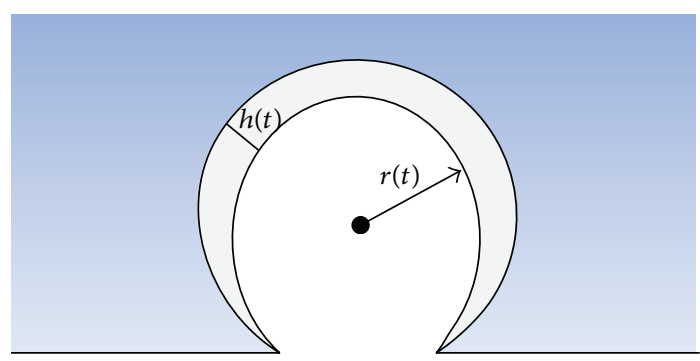

FIgURE 2: Schema of spherical parenchymal lesion. A schematic drawing of the spherical membrane geometry used as the basis for studying the growth of axisymmetric lung parenchymal lesions.

If the $2 \mathrm{D}$ deformation gradient $\mathbf{F}$ is $\operatorname{diag}(\lambda(t), \lambda(t))$, then the Cauchy stress resultant tensor $\mathbf{T}$ for inner membrane stress can be represented by the following equation [11]:

$$
T_{\alpha \beta}=\frac{1}{\operatorname{det} \mathbf{F}} F_{\alpha \gamma} F_{\beta \delta} \frac{\partial w}{\partial E_{\gamma \delta}}+\frac{2 \mu_{m} H}{\lambda(t)^{3}} \frac{d \lambda(t)}{d t}, \quad \alpha, \beta, \gamma, \delta=1,2 .
$$

Thus, the isotropic stress resultant $T\left(T_{11}\right)$ is given by the following:

$$
\begin{aligned}
T= & -\frac{c_{1} H}{c_{2}}+c_{3} H+\left(-\frac{c_{1} H}{2 c_{2}^{2}}+\frac{3 c_{1} H}{2 c_{2}}-2 c_{3} H\right)\left(\lambda(t)^{2}-1\right) \\
& +\frac{2 \mu_{m} H}{\lambda(t)^{3}} \frac{d \lambda(t)}{d t} .
\end{aligned}
$$

2.1.2. Model for Breathing Motion. We used a Fourier series developed by Jakuszkin [12] to model the pulsatile nature of breathing. The driven flow value of air through the lungs $Q$ is

$$
Q=\sum_{i=1}^{7} A_{i} \cos \left(2 \pi f_{i} t+\phi_{i}\right) .
$$

Table 1 contains the constants $A_{i}, f_{i}$, and $\phi_{i}[12]$.

Note that while we have employed a sinusoidal model for simplicity, we can extend the model to account for erratic breathing behavior, which is not considered here. The driven flow value is related to the pulsatile pressure through the relation $\Delta P=R_{b} Q$, where $R_{b}$ was the respiratory resistance of the bronchiole network. Because $R_{b}$ is $1.0 \mathrm{mmHg} \cdot \mathrm{s} \cdot \mathrm{L}^{-1}$ [13] and the original pressure was atmospheric pressure (760 $\mathrm{mmHg}$ ), the final pressure series was as follows:

$$
P_{\text {inner }}=760+\sum_{i=1}^{7} A_{i} \cos \left(2 \pi f_{i} t+\phi_{i}\right) .
$$

2.1.3. Dynamic Equation System. We obtained the dynamic equation for the wall through linear momentum balance to yield

$$
\rho_{m} h R \frac{d^{2} \lambda(t)}{d t^{2}}=P(t)-\frac{2 T(\lambda(t))}{\lambda(t) R},
$$

TABLE 1: Lung Fourier series parameter table.

\begin{tabular}{cccc}
\hline$i$ & $A\left(\mathrm{~L} \cdot \mathrm{s}^{-1}\right)$ & $f(\mathrm{~Hz})$ & $\phi$ \\
\hline 1 & 1 & 0.156250 & 4.95 \\
2 & 0.3 & 0.390625 & 3.82 \\
3 & 0.25 & 0.859375 & 4.37 \\
4 & 0.25 & 1.484375 & 3.67 \\
5 & 0.175 & 2.421875 & 4.05 \\
6 & 0.175 & 4.609375 & 4.13 \\
7 & 0.125 & 8.046875 & 4.02 \\
\hline
\end{tabular}

where $\rho_{m}$ is the density of the membrane and $P(t)$ is the inner pressure minus the outer pressure (transmural pressure). The Navier-Stokes equations in spherical coordinates were used to model the pressure that the pleural fluid exerted on the lung parenchyma. The resultant pressure was as follows [14]:

$$
P_{\text {outer }}=p_{\infty}+\rho_{f} R^{2}\left(\lambda(t) \frac{d^{2} \lambda(t)}{d t^{2}}+\frac{3}{2}\left(\frac{d \lambda(t)}{d t}\right)^{2}\right),
$$

where $p_{\infty}$ is a constant value representing the pressure at a significantly large distance from the lung and $\rho_{f}$ is the density of the fluid. Note the absence of a viscous term as well as the same convention for the pressure at infinity as in the published work by Shah and Humphrey [14]. Combining the equations for the dynamic wall, the outer pressure, the dynamic radial stress which contributes the factor of $\mu_{f}$, and viscoelasticity, we arrived at the following differential equation for the air-tissue-pleural fluid system:

$$
\begin{gathered}
\left(\frac{\rho_{m} H R}{\lambda(t)^{2}}+\rho_{f} R^{2} \lambda(t)\right) \frac{d^{2} \lambda(t)}{d t^{2}}+\frac{3}{2} \rho_{f} R^{2}\left(\frac{d \lambda(t)}{d t}\right)^{2}+4 \frac{\mu_{f}}{\lambda(t)} \\
\times \frac{d \lambda(t)}{d t}+\frac{2 T(\lambda(t))}{\lambda(t) R}+\frac{4 \mu_{m} H}{\lambda(t)^{4} R} \frac{d \lambda(t)}{d t}=P_{\text {inner }}(t)-p_{\infty},
\end{gathered}
$$

where $\mu_{f}$ and $\mu_{m}$ are the dynamic viscosities of the pleural fluid and membrane, respectively.

2.1.4. Nondimensionalization. Nondimensionalization is a process that helps to accommodate multiscale variations in the data. This technique is a common engineering practice which essentially simplifies multiphysics problems with different measured units involved. Because our final governing equations couple three different physical aspects (the air, the membrane, and the pleural fluid), this method was necessitated. Moreover, nondimensionalization recovers the characteristic properties of the stretch ratio and stretch rate. We followed this standard which is commonly used in published 
TABLE 2: List of parameters.

\begin{tabular}{lcc}
\hline Parameter (variable) & Value & Citation \\
\hline Material parameters $\left(c_{1}, c_{2}, c_{3}\right)$ & $-22.5 \times 10^{5} \mathrm{~N} \cdot \mathrm{m}^{-2}, 1.26,-7.8 \times 10^{5} \mathrm{~N} \cdot \mathrm{m}^{-2}$ & Denny and Schroter, 2006 [8] \\
Undeformed lesion thickness $(H)$ & $10^{-3} \mathrm{~m}$ & Amjadi et al., 2007 [15] \\
Undeformed lesion radius $(R)$ & $10^{-2} \mathrm{~m}$ & Amjadi et al., 2007 [15] \\
Resistance of bronchiole network $\left(R_{b}\right)$ & $1 \mathrm{mmHg} \cdot \mathrm{s} \cdot \mathrm{L}^{-1}$ & Ben-Tal, 2006 [13] \\
Pressure at infinity $\left(p_{\infty}\right)$ & $0 \mathrm{mmHg}$ & Imposed \\
Pleural fluid viscosity $\left(\mu_{f}\right)$ & $1.39-1.57 \times 10^{-3} \mathrm{~Pa} \cdot \mathrm{s}$ & Yetkin et al., 2007 [16] \\
Pleural fluid density $\left(\rho_{f}\right)$ & $980.3 \mathrm{~kg} \cdot \mathrm{m}^{-3}$ & Rubins and Manning, "Pleural Effusion Workup" [17] \\
Solid membrane viscosity $\left(\mu_{m}\right)$ & $7 \times 10^{-2} \mathrm{~Pa} \cdot \mathrm{s}$ & Girnyk et al., 2006 [18] \\
Membrane density $\left(\rho_{m}\right)$ & $1050 \mathrm{~kg} \cdot \mathrm{m}^{-2}$ & Shah and Humphrey, 1999 [14] \\
Atmospheric pressure & $760 \mathrm{mmHg}$ & Ben-Tal, 2006 [13]
\end{tabular}

works on biomechanics such as Shah and Humphrey [14]. The following nondimensionalized quantities were required:

$$
\begin{gathered}
y_{0} \equiv \lambda(t), \quad \tau \equiv t \sqrt{\frac{\left|c_{1}\right| H}{\rho_{m} R^{2} H}}, \quad b \equiv \frac{\rho_{f} R}{\rho_{m} H}, \\
d \equiv \frac{H}{R}, \quad m_{f} \equiv \frac{\mu_{f}}{\sqrt{\rho_{m}\left|c_{1}\right| H^{2}}}, \quad m \equiv \frac{\mu_{m}}{\sqrt{\rho_{m}\left|c_{1}\right| H^{2}}}, \\
F(\tau) \equiv\left(P_{\text {inner }}(t)-p_{\infty}\right) \frac{R}{c_{1} H}, \quad f \equiv \frac{T}{c_{1} H} .
\end{gathered}
$$

The resulting nondimensionalized equation system was as follows:

$$
\begin{gathered}
\frac{d y_{0}}{d \tau}=y_{1}, \\
\frac{d y_{1}}{d \tau} \\
=\frac{F(\tau)-3 b y_{1}^{2} / 2-4 m_{f} y_{1} / y_{0}-2 f\left(y_{0}\right) / y_{0}-4 m d y_{1} / y_{0}^{4}}{b y_{0}+y_{0}^{-2}} .
\end{gathered}
$$

2.1.5. Parameter Table. Table 2 is a complete collection of the relevant parameter values for the model.

2.2. Initial Numerical Investigations. We conducted several numerical investigations in order to quantify both system dynamics and stability. The previous dynamical system was numerically evolved in MATLAB through a fourth-order explicit Runge-Kutta scheme. Plots of the stretch ratio $\lambda(t)$ versus time reveal sustained stable oscillations of the membrane for normal lung parenchymal tissue parameters of the Denny-Schroter model: $c_{1}=-22.5 \times 10^{5}, c_{2}=1.26$, and $c_{3}=$ $-7.8 \times 10^{5}$. Variations in material stiffness proved an effective tool in analyzing the effects of parameter variation on system dynamics. Figure 3 shows that as material stiffness increased in the model (for test values of $c_{2}=1.00,1.26$, and 1.52), the amplitude of the oscillations decreased as one would expect intuitively. Figure 4 shows that for the general range of $c_{2} \in$ $[.5,1.6]$, amplitude can be seen to monotonically decrease and

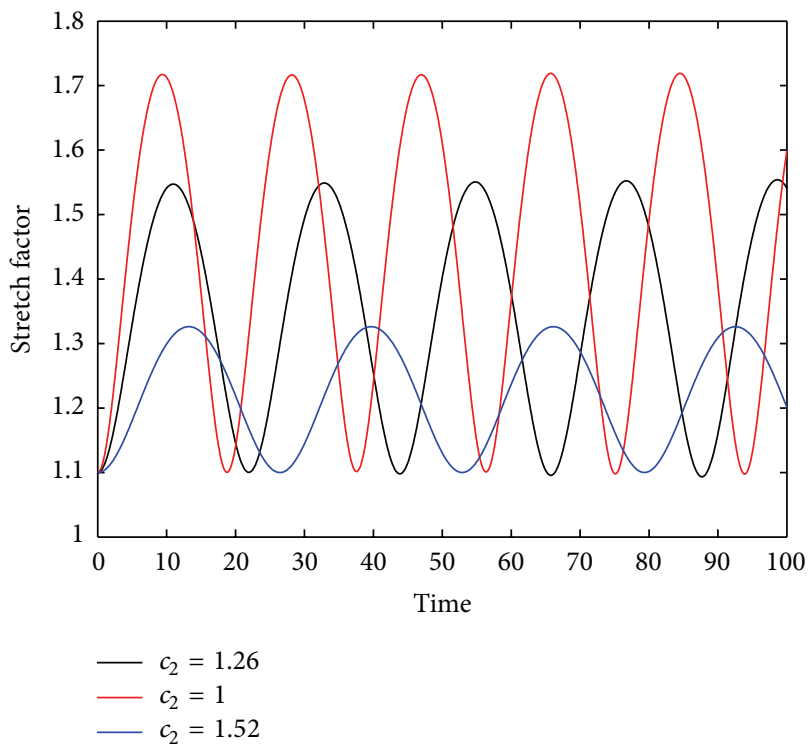

FIGURE 3: Stretch factor $\lambda(t)$ for different stiffness constant $\left(c_{2}\right)$. This figure shows three plots of stretch factor with respect to dimensionless time for the stiffness constants $c_{2}=1.00,1.26$, and 1.52. Variations in the stiffness constant clearly affect the amplitude and frequency of oscillations.

begin to level off near zero. For the same window of stiffness constants, oscillation frequency reaches a peak near $c_{2} \approx 0.78$ and then decreases monotonically from that value.

Figure 5 demonstrates that the system is stable even with minor perturbations in initial conditions for that class of material parameters. This suggests that there may be conditions where the bleb will not rupture. Figure 6 is explained in the preceding paragraph as a relative force proportion graph, which illustrates the dynamic behavior of the different forces including internal pressure, fluid structure, internal membrane, radial stress, and viscoelasticity. The graph suggests that friction is not a significant factor leading to pneumothorax, and this aligns with other published works. The numerical phase plane analysis in Figure 5 of $\lambda(t)$ versus $\dot{\lambda}(t)$ further suggests the existence of an orbit about a stable equilibria. The spiral nature of the superimposed vector field, representing the stretch rate $\dot{\lambda}(t)$ versus the derivative of the 


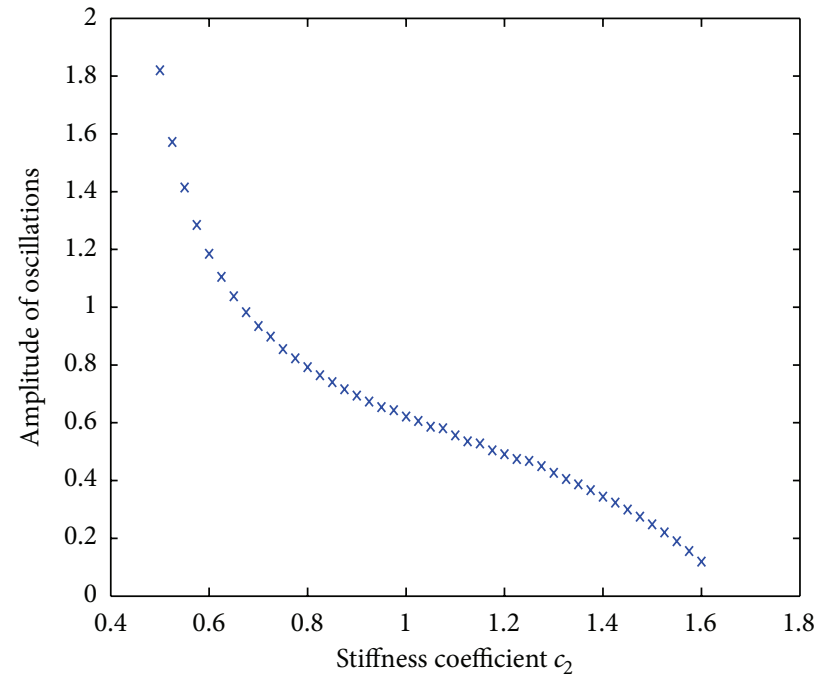

(a)

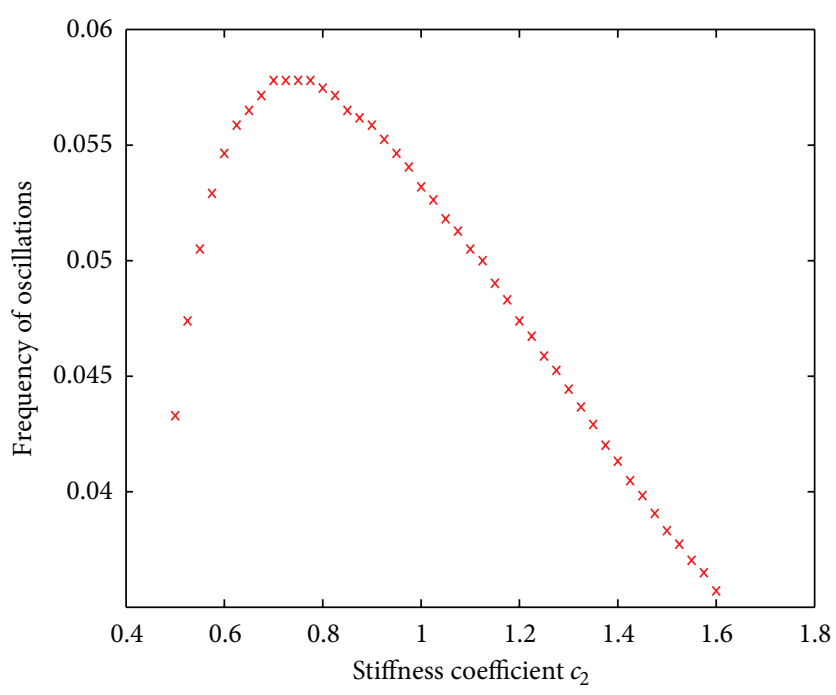

(b)

FIGURE 4: Quantified effects of stiffness constant $\left(c_{2}\right)$ on amplitude and frequency of oscillations. (a) The graph shows the effect of variations in stiffness constant on the amplitude of oscillations. The monotonically decreasing nature of the graph suggests that the stiffer the membrane, the smaller the amplitude. (b) The graph shows the effect of variations in stiffness constant on the frequency of oscillations with a maximum at $c_{2} \approx 0.78$.

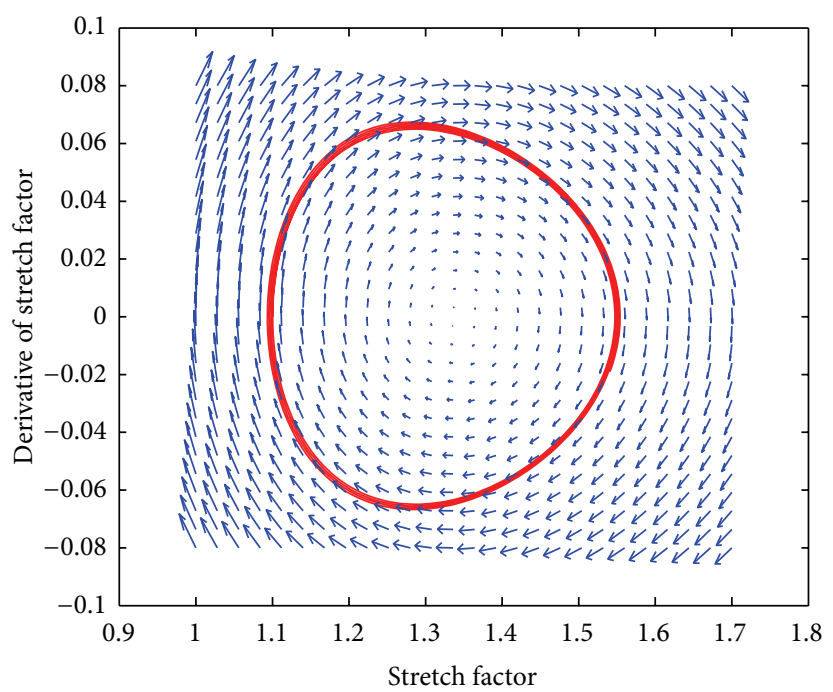

(a)

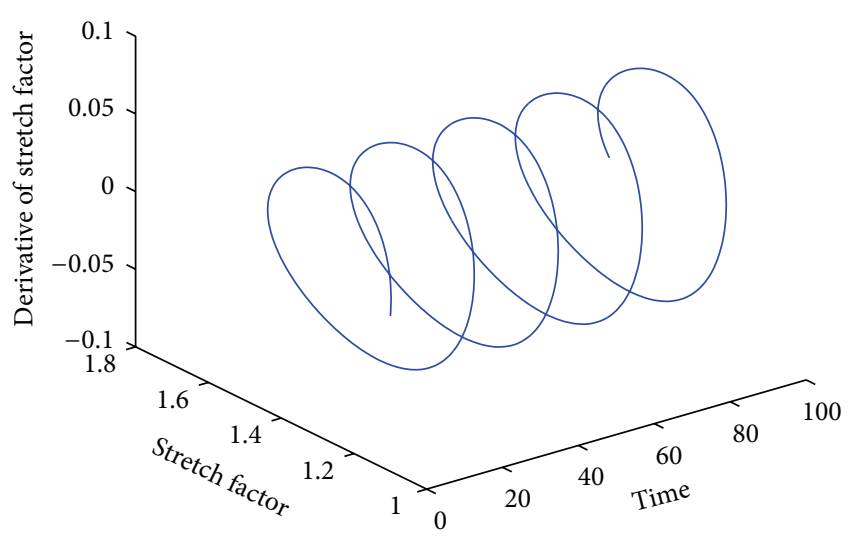

(b)

FiguRE 5: Numerically generated phase planes of the dynamical system. (a) The stable orbit and superimposed vector field demonstrated in the phase plane suggest the presence of an asymptotically stable equilibrium. (b) The $3 \mathrm{D}$ plot depicts the evolution of the stable orbit with respect to dimensionless time as further evidence of sustained stable oscillations. Refer to Section 2.4 for more information about physically unstable situations.

stretch rate $\ddot{\lambda}(t)$, suggests an asymptotically stable node at $\lambda_{\mathrm{eq}} \approx 1.3$ for normal lung parenchymal tissue parameters. A relative force proportion graph in Figure 6 reveals the forces of internal pressure and inner membrane stress as the major driving forces behind this oscillation, whereas the viscoelastic and fluid forces effects are negligible. Furthermore, several unstable regions had been isolated through variation of the material parameters such as for $c_{1}=-22.5 \times 10^{5}, c_{2}=1.26$, and $c_{3}=-1.39 \times 10^{6}$, but a rigorous mathematical analysis had been called for to fully determine whether the instabilities were truly physical or just due to numerical effects.

\subsection{Stability Results}

2.3.1. Determining System Equilibria. In order to simplify the following equilibria identification and stability analysis, we made the system autonomous by approximating $F(t) \approx$ $F=R P / c_{1} H$. The system is at an equilibrium for any point 


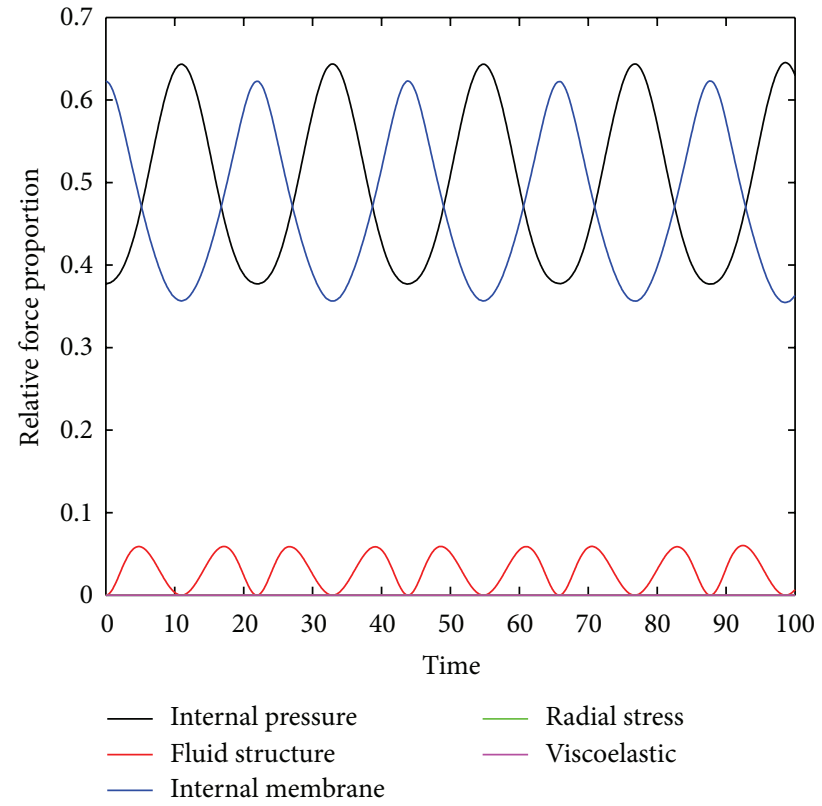

(a)

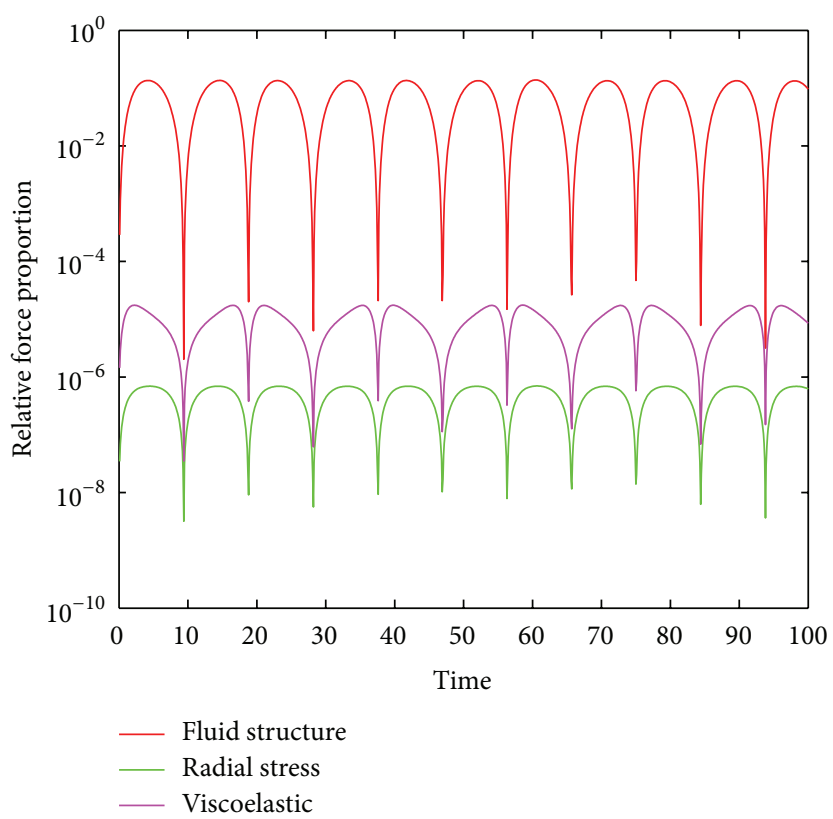

(b)

FIGURE 6: Relative force proportion graph. (a) The graph depicts the relative magnitude of the dimensionless forces at play over dimensionless time: pressure $(F(\tau))$, radial stress $\left(4 m_{f} y_{1} / y_{0}\right)$, internal membrane $\left(2 f\left(y_{0}\right) / y_{0}\right)$, fluid structure $\left(3 b y_{1}^{2} / 2\right)$, and viscoelastic $\left(4 m d y_{1} / y_{0}\right)$. It is clear that internal pressure and internal membrane forces are the major driving forces behind the oscillations. (b) The three forces that contribute the least (radial stress, viscoelastic, and fluid structure) are plotted on semilogarithmic axes.

$(\lambda(t), \dot{\lambda}(t))$ such that $\dot{y}_{0}=0$ and $\dot{y}_{1}=0$. This implies that $\dot{\lambda}(t)=0$ for any equilibrium point. The equilibrium stretch ratio $\lambda_{\text {eq }}$ may be found by solving $F-(f(\lambda(t)) / \lambda(t))=0$ as follows:

$$
\lambda_{\text {eq }}=\frac{-\alpha \pm \sqrt{-4 \beta \gamma+\alpha^{2}}}{2 \gamma},
$$

where $\alpha=c_{1} c_{2}^{2} F, \beta=-c_{1}+5 c_{1} c_{2}-6 c_{2}^{2} c_{3}$, and $\gamma=c_{1}-$ $3 c_{1} c_{2}+4 c_{2}^{2} c_{3}$. For physically meaningful values of $\lambda(t)$, the positive equilibrium point is the one of significance. For the normal lung parenchymal tissue parameters, $\lambda_{\mathrm{eq}}^{+}=1.3405$ as suggested by the initial numerical investigation.

2.3.2. Characterization of Unstable Nodes. We conducted a general ordinary differential equation (ODE) stability analysis on the physical system in order to evaluate a lung parenchymal lesion with arbitrary material parameters for physical instabilities. Given a system with Jacobian matrix $J$, ODE instability is prescribed for det $J<0$ or $\operatorname{tr} J>0$. For the previous dynamical system in $y_{0}$ and $y_{1}$, the Jacobian matrix about the positive equilibrium point reduces to

$$
J=\left[\begin{array}{cc}
0 & 1 \\
\frac{2 F \gamma\left(-4 \beta \gamma+\alpha^{2}-\alpha \sqrt{-4 \beta \gamma+\alpha^{2}}\right)}{\alpha\left(-\alpha+\sqrt{-4 \beta \gamma+\alpha^{2}}\right)^{2}\left(\left(4 \gamma^{2} /\left(-\alpha+\sqrt{-4 \beta \gamma+\alpha^{2}}\right)^{2}\right)+\left(b\left(-\alpha+\sqrt{-4 \beta \gamma+\alpha^{2}}\right) / 2 \gamma\right)\right)} & \frac{\left(2 m_{f}\left(\alpha+\sqrt{-4 \beta \gamma+\alpha^{2}}\right) / \beta\right)-\left(64 \gamma^{4} d m /\left(-\alpha+\sqrt{-4 \beta \gamma+\alpha^{2}}\right)^{4}\right)}{\left(\left(4 \gamma^{2} /\left(-\alpha+\sqrt{-4 \beta \gamma+\alpha^{2}}\right)^{2}\right)+\left(b\left(-\alpha+\sqrt{-4 \beta \gamma+\alpha^{2}}\right) / 2 \gamma\right)\right)}
\end{array}\right] .
$$

The constraint of physically relevant material parameters imposes the inequalities $c_{1}<0, c_{2}>0$, and $c_{3}<0$, whereas the constraint of defined real elements of the Jacobian matrix imposes $\beta \neq 0, \gamma \neq 0$, and $\alpha^{2} \geq 4 \beta \gamma$. Further note that from the definition of $F=A P / c_{1} H, \alpha$ is always greater than zero for physically relevant material constants $(A, H, P>0)$.
Theorem 1. For any values of $\gamma$ and $\beta$ such that $\gamma<0, \beta>0$, and $\gamma<b^{1 / 3} \alpha-b^{2 / 3} \beta$, the system is unstable because $\operatorname{det} J<0$.

The expression det $J=-\partial \dot{y}_{1} / \partial y_{0}$ is less than zero for all $\beta, \gamma$ such that $\operatorname{sgn}\left(\partial \dot{y}_{1} / \partial y_{0}\right)>0$. The sign of this expression is in turn governed by the following three 
subexpressions. The subexpression of $\left(-\alpha+\sqrt{-4 \beta \gamma+\alpha^{2}}\right)^{2}$ is consciously excluded from the sign analysis because it is invariantly positive.

Lemma 2. The sign of $\beta$ and $\gamma$ cannot both be positive for physically relevant parameters.

The lemma is proven by contradiction. By definition, $\beta>0$ implies $-c_{1}+5 c_{1} c_{2}-6 c_{2}^{2} c_{3}>0$. Isolation of $c_{3}$ reduces the inequality to $c_{3}<c_{1}\left(5 c_{2}-1\right) / 6 c_{2}^{2}$. Likewise, $\gamma=c_{1}-3 c_{1} c_{2}+$ $4 c_{2}^{2} c_{3}>0$ reduces to $c_{3}>c_{1}\left(3 c_{2}-1\right) / 4 c_{2}^{2}$ and places bounds on $c_{3}$. However, the inequality $c_{1}\left(3 c_{2}-1\right) / 4 c_{2}^{2}<c_{1}\left(5 c_{2}-1\right) / 6 c_{2}^{2}$ reduces to $0<c_{1}\left(c_{2}+1\right)$, which cannot be true from the restrictions of $c_{1}<0$ and $c_{2}>0$.

Lemma 3. The expression

$$
-4 \beta \gamma+\alpha^{2}-\alpha \sqrt{-4 \beta \gamma+\alpha^{2}}
$$

is greater than zero for any value of $\beta, \gamma$ such that either $\beta<0$ and $\gamma>0$ or $\beta>0$ and $\gamma<0$. Similarly, the expression is less than zero for any value of $\beta, \gamma$ such that either $\beta<0$ and $\gamma<0$ or $\beta>0$ and $\gamma>0$.

The inequality $-4 \beta \gamma+\alpha^{2}-\alpha \sqrt{\alpha^{2}-4 \beta \gamma}>0$ reduces to $\alpha^{2}-$ $4 \beta \gamma>\alpha \sqrt{\alpha^{2}-4 \beta \gamma}$. Because both sides of the inequality are positive, the inequality may be squared and the terms brought to one side to give $\left(\alpha^{2}-4 \beta \gamma\right)(-4 \beta \gamma)>0$. Because only values of $\alpha, \beta$, and $\gamma$ such that $\alpha^{2}-4 \beta \gamma>0$ are considered, the expression is true $\beta \gamma<0$. Thus, $-4 \beta \gamma+\alpha^{2}-\alpha \sqrt{-4 \beta \gamma+\alpha^{2}}>0$ for either $\beta<0$ and $\gamma>0$ or $\beta>0$ and $\gamma<0$. Likewise, $-4 \beta \gamma+\alpha^{2}-\alpha \sqrt{-4 \beta \gamma+\alpha^{2}}<0$ for $\beta<0$ and $\gamma<0$ or $\beta>0$ and $\gamma>0$.

Lemma 4. For physically relevant restriction of $b>0$, the expression

$$
\frac{4 \gamma^{2}}{\left(-\alpha+\sqrt{-4 \beta \gamma+\alpha^{2}}\right)^{2}}+\frac{b\left(-\alpha+\sqrt{-4 \beta \gamma+\alpha^{2}}\right)}{2 \gamma}
$$

is greater than zero for any value of $\beta, \gamma$ such that any one of the following three conditions are met: $\gamma>0$ and $\beta<0 ; \gamma<0$ and $\beta<0 ; \gamma<b^{1 / 3} \alpha-b^{2 / 3} \beta, \gamma<0$, and $\beta>0$.

For either the conditions of $\gamma>0$ and $\beta<0$ or $\gamma<0$ and $\beta<0$, the expression $b\left(-\alpha+\sqrt{-4 \beta \gamma+\alpha^{2}}\right) / 2 \gamma$ is always greater than zero. Hence, because $4 \gamma^{2} /\left(-\alpha+\sqrt{-4 \beta \gamma+\alpha^{2}}\right)$ is invariantly positive, the entire expression (15) will be greater than zero for these conditions. Given $\gamma, \beta$ such that $\gamma<0$ and $\beta>0$, the inequality reduces to $8 \gamma^{3}+b\left(-\alpha+\sqrt{-4 \beta \gamma+\alpha^{2}}\right)<0$ or $\gamma<-\left(b^{1 / 3} / 2\right)\left(-\alpha+\sqrt{-4 \beta \gamma+\alpha^{2}}\right)$. This in turn reduces to $4 \gamma / b^{2 / 3}-4 \alpha / b^{1 / 3}+4 \beta<0$ or $\gamma<b^{1 / 3} \alpha-b^{2 / 3} \beta$.
Lemma 5. For physically relevant restriction of $b>0$, the expression (15) is less than zero for any value of $\beta, \gamma$ such that $\gamma<0, \beta>0$, and $\gamma>b^{1 / 3} \alpha-b^{2 / 3} \beta$.

Either the conditions of $\gamma>0$ and $\beta<0$ or $\gamma<0$ and $\beta<0$ make the statement unconditionally false, leaving only the case of $\gamma<0, \beta>0$. The fact that $\gamma>b^{1 / 3} \alpha-b^{2 / 3} \beta$ is proven likewise as in Lemma 4.

Lemma 6. The expression

$$
\frac{2 F \gamma}{\alpha}
$$

is greater than zero for all $\gamma<0$ and less than zero for all $\gamma>0$. By definition, $F<0$ and $\alpha>0$, and thus the result follows.

The expression det $J<0$ is true such that the product of expressions (14), (15), and (16) is positive. Upon coupling of the previous inequality systems, the only scenario that does not lead to contradictions is for (14), (15), and (16) all positive. This case implies that for $\gamma<0, \beta>0$, and $\gamma<b^{1 / 3} \alpha-b^{2 / 3} \beta$ the system is unstable because det $J<0$.

Theorem 7. For any values of $\gamma$ and $\beta$ such that $\gamma<0, \beta>0$, $\gamma>b^{1 / 3} \alpha-b^{2 / 3} \beta$, and $\beta>\alpha \gamma^{2}\left(d m / m_{f}\right)^{1 / 3}+\gamma\left(d m / m_{f}\right)^{2 / 3}$, the system is unstable because $\operatorname{tr} J>0$.

The expression $\operatorname{tr} J=-\partial \dot{y}_{1} / \partial y_{1}$ is less than zero for all $\beta, \gamma$ such that $\operatorname{sgn}\left(\partial \dot{y}_{1} / \partial y_{1}\right)>0$. The sign of this expression is in turn governed by subexpressions (15) and (17).

Lemma 8. For physically relevant parameters $\left(m_{f}, m, d>0\right)$,

$$
\frac{2 m_{f}\left(\alpha+\sqrt{-4 \beta \gamma+\alpha^{2}}\right)}{\beta}-\frac{64 \gamma^{4} d m}{\left(-\alpha+\sqrt{-4 \beta \gamma+\alpha^{2}}\right)^{4}}
$$

is less than zero for all $\beta<0$ or for $\beta>0, \gamma<0$, and $\beta>$ $\alpha \gamma^{2}\left(d m / m_{f}\right)^{1 / 3}+\gamma\left(d m / m_{f}\right)^{2 / 3}$.

For all $\beta<0$, expression (17) is invariantly negative, and thus, the statements are unconditionally true. This leaves only the case of $\beta>0$ and $\gamma<0$. Manipulation of the expression yields

$$
\frac{2 m_{f}}{\beta}\left(\alpha+\sqrt{\alpha^{2}-4 \beta \gamma}\right)\left(-\alpha+\sqrt{\alpha^{2}-4 \beta \gamma}\right)^{4}<64 \gamma^{4} d m
$$

or $\left(-\alpha+{\sqrt{\alpha^{2}-4 \beta \gamma}}^{3}>-8 \gamma^{3} d m / m_{f}\right.$. This in turn yields $\alpha^{2}-$ $4 \beta \gamma>\left(\alpha-2 \gamma\left(d m / m_{f}\right)^{1 / 3}\right)^{2}$, which upon isolation of $\beta$ gives the expression $\beta>\alpha \gamma^{2}\left(d m / m_{f}\right)^{1 / 3}+\gamma\left(d m / m_{f}\right)^{2 / 3}$.

The expression $\operatorname{tr} J>0$ is true such that the product of expressions (15) and (17) is positive. For the signs of (15) and (17) to both be negative, Lemma 5 and Lemma 8 give $\gamma<0, \beta>0, \gamma>b^{1 / 3} \alpha-b^{2 / 3} \beta$, and $\beta>$ $\alpha \gamma^{2}\left(d m / m_{f}\right)^{1 / 3}+\gamma\left(d m / m_{f}\right)^{2 / 3}$. The case of expression (15) and (17) both greater than zero is consciously omitted from 


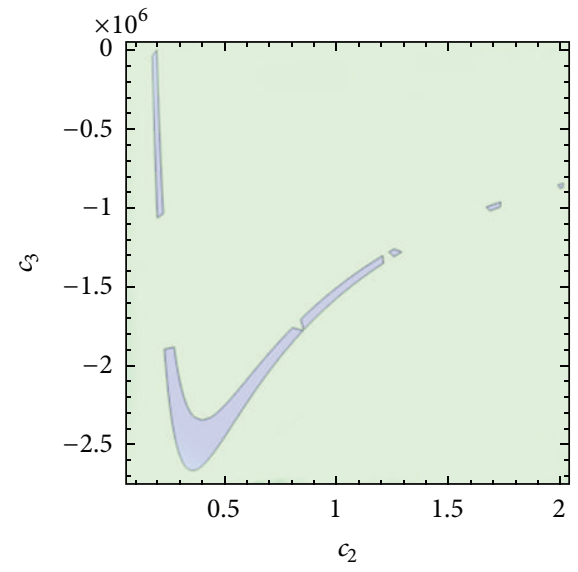

(a)

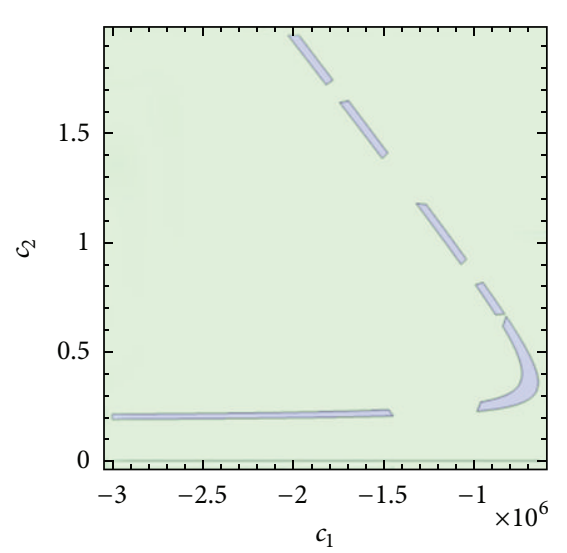

(b)

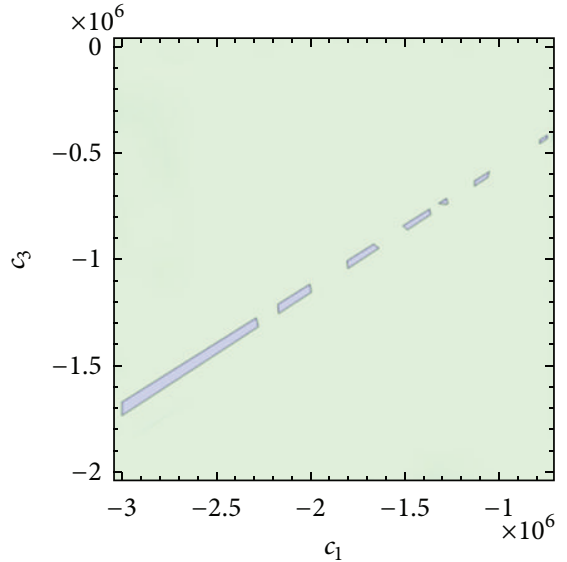

(c)

FIGURE 7: 2D instability region portraits. The images show the regions of instability (blue) for each selection of two material parameters. The value of the third parameter in each graph is assumed to be the value from Denny and Schroter [8].

the proof because the analysis leads to an instability region already enclosed by Theorem 1 . Figure 7 shows $2 \mathrm{D}$ portraits of the regions obtained from these instability criteria for a certain selection of material parameters $c_{1}, c_{2}$, and $c_{3}$.

2.4. Introducing Energy Limitations of Tissue. In order to accurately capture the biomechanics of lung parenchymal failure, we translated ODE instabilities to physical mechanical failure via the introduction of a finite energy limiter $\Phi\left(\mathrm{Jm}^{-3}\right)$. Similar to Volokh and Vorp [19], we built a finite energy constitutive model $\Psi$ based on the framework of the hyperelastic model $w$ which was constructed as follows:

$$
\Psi=H \Phi\left(1-\exp \left(\frac{-w}{H \Phi}\right)\right)
$$

Note that as $w \rightarrow \infty, \Psi \rightarrow H \Phi$ a finite value of energy before rupture in contrast to the hyperelastic model where $w$ may grow unbounded as $\lambda(t) \rightarrow \infty$. Similarly, in the limiting case $H \Phi \ll w, \Psi \approx w$ as can be seen via Taylor expansion. Reformulating the Cauchy stress resultant tensor $\mathbf{T}$ given by [14] with the new strain-energy function yields

$$
T_{\alpha \beta}^{\prime}=\frac{1}{\operatorname{det} \mathbf{F}} F_{\alpha \gamma} F_{\beta \delta} \frac{\partial \Psi}{\partial E_{\gamma \delta}}+\frac{2 \mu_{m} H}{\lambda(t)^{3}} \frac{d \lambda(t)}{d t}, \quad \alpha, \beta, \gamma, \delta=1,2 .
$$

Thus, the new isotropic stress resultant $T^{\prime}\left(T_{11}^{\prime}\right)$ is given by the following:

$T^{\prime}$

$$
=\left(-\frac{c_{1} H}{c_{2}}+c_{3} H+\left(-\frac{c_{1} H}{2 c_{2}^{2}}+\frac{3 c_{1} H}{2 c_{2}}-2 c_{3} H\right)\left(\lambda(t)^{2}-1\right)\right)
$$

$$
\begin{aligned}
& \quad \times \exp \left(\frac{\left(c_{1} H / c_{2}-c_{3} H\right)\left(\left(\lambda(t)^{2}-1\right) / 2\right)}{H \Phi}\right. \\
& \left.+\frac{\left(c_{1} H / 2 c_{2}^{2}-3 c_{1} H / 2 c_{2}+2 c_{3} H\right)\left(\left(\lambda(t)^{2}-1\right)^{2} / 4\right)}{H \Phi}\right) \\
& +\frac{2 \mu_{m} H}{\lambda(t)^{3}} \frac{d \lambda(t)}{d t}
\end{aligned}
$$

As demonstrated in Figure 8, numerical simulations with the new isotropic stress resultant demonstrate rupture midexpansion as opposed to expansion to infinity in the original constitutive model.

2.5. Collagen-Elastin Dynamics. The final component of the proposed model for parenchymal lesion growth and rupture is a means by which stable mechanical configurations may enter unstable domains. The introduction of collagen-elastin dynamics (a dynamic protein framework of the tissue) elucidates mechanisms for how a stable lesion may eventually rupture. We introduced a new constitutive model through the creation of a linear combination of Denny and Schroter's [8] collagen-only strain energy function and Humphrey and Yin's [20] elastin-only strain energy function:

$$
\begin{aligned}
w^{\prime}= & A_{c}(\tau)\left(\frac{H}{\lambda(t)^{2}}\left(c_{1} \log \left[1-\left(\frac{e^{E_{11}}-1}{c_{2}}\right)\right]+c_{3} E_{11}\right)\right) \\
& +A_{e}(\tau)\left(\frac{b H}{\lambda(t)^{2}}(\lambda(t)-\log \lambda(t)-1)\right) \\
w^{\prime} \approx & \left(\frac{-A_{c}(\tau) c_{1} H}{c_{2}}+A_{c}(\tau) c_{3} H\right) \frac{\lambda(t)^{2}-1}{2}
\end{aligned}
$$




$$
\begin{gathered}
+\left(\frac{A_{e}(\tau) b H}{2}-\frac{A_{c}(\tau) c_{1} H}{2 c_{2}^{2}}+\frac{3 A_{c}(\tau) c_{1} H}{2 c_{2}}\right. \\
\left.-2 A_{c}(\tau) c_{3} H\right) \frac{\left(\lambda(t)^{2}-1\right)^{2}}{4},
\end{gathered}
$$

where $A_{c}(\tau)$ and $A_{e}(\tau)$ are dimensionless quantities representing active collagen and elastin numbers. Similarly as before, we introduced a finite energy limiter $\Psi^{\prime}$ to this constitutive model, and we recalculated the new isotropic strain tensor. Unlike the previous developments of the paper, collagen-elastin dynamics called for a return to the initial momentum balance due to the changing mass of the protein matrix. Given the mass of collagen $C$ and elastin $E$ defined by $\rho_{c} A_{c}$ and $\rho_{e} A_{e}$ (where $\rho_{c}$ and $\rho_{e}$ are equal to $5.10 \mathrm{~kg} \cdot \mathrm{m}^{-3}$ [21]), the new momentum balance was as follows:

$$
\rho_{m} h R \frac{d^{2} \lambda(t)}{d t^{2}}+\frac{d\left(A_{e} \rho_{e}+A_{c} \rho_{c}\right)}{d t} v_{r} H=P(t)-\frac{2 T(\lambda(t))}{\lambda(t) R},
$$

where $v_{r}(t)$ is the velocity at the boundary of an expanding biological membrane given by $R d \lambda(t) / d t$. With the nondimensionalized term $m_{p}=\left(A / \sqrt{\rho_{m}\left|c_{1}\right|}\right)(d C / d t+d E / d t)$, the first two differential equations of the dynamical system became

$$
\begin{gathered}
\frac{d y_{0}}{d \tau}=y_{1}, \\
\frac{d y_{1}}{d \tau} \\
=\frac{F(\tau)-3 b y_{0}^{2} / 2-4 m_{f} y_{1} / y_{0}-2 f\left(y_{0}\right) / y_{0}-4 m d y_{1} / y_{0}^{4}-m_{p} y_{1} / y_{0}^{2}}{b y_{0}+y_{0}^{-2}} .
\end{gathered}
$$

Based on histochemical evidence that had determined that collagen levels increase with increased stress and that elastin levels decrease because of elastolytic processes near rupture [22], we proposed the following differential equations for collagen-elastin dynamics:

$$
\frac{d A_{c}}{d t}=\frac{k_{1} A_{c}}{\left|\phi^{\prime}-\psi^{\prime}\right|^{n}}, \quad \frac{d A_{e}}{d t}=\frac{-k_{2} A_{e}}{\left|\phi^{\prime}-\psi^{\prime}\right|^{n}} .
$$

Because $k_{1}$ and $k_{2}$ are positive constants, we devised the differential equations such that little change occurs in the protein matrix in scenarios far from rupture $\left(\left|\phi^{\prime}-\psi^{\prime}\right| \gg 0\right)$ and larger changes occur as the membrane nears rupture. We numerically solved the series of four differential equations as a whole in MATLAB, revealing escapes from stable orbits for certain rate kinetics of the elastin-collagen dynamics that are depicted in Figure 9.

2.6. Parameter Estimation from In Vivo Data. In order to build a database for parameter estimation on the proposed model, we collected in vivo lung data in the form of CT scans from the VIA/I-ELCAP Public Lung Image Database, which

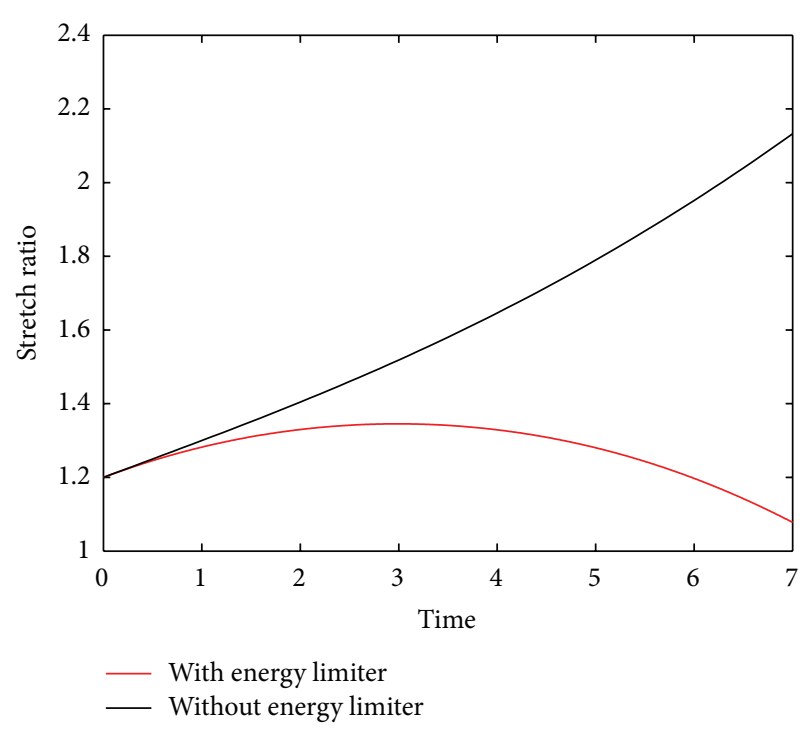

FIGURE 8: Effect of introduction of finite energy limiter. A point $\left(c_{1}, c_{2}, c_{3}\right)$ which satisfies the instability of requirement of Theorem $1\left(-22.5 \times 10^{5}, 1.26\right.$, and $\left.-1.39 \times 10^{6}\right)$ was used to test the effect of the finite energy limiter. As indicated by the red plot, the finite energy limiter curtails expansion to infinity and establishes a physical rupture point.

were about $250 \mathrm{~mm} \times 150 \mathrm{~mm}$ in size. The Sobel Edge Detection algorithm provided a means to quantify the deformation of the lung boundary and to extract material parameters from in vivo clinical data for the strain-energy function. This algorithm works by estimating the horizontal and vertical spatial gradients at the interior pixels, $G_{x}$ and $G_{y}$, through application of a matrix mask in order to detect edges. Pixels that meet a certain threshold for $G\left(\left|G_{x}\right|+\left|G_{y}\right|\right)$ are counted as edges (Aybar, "Sobel Edge Detection"). This method was preferred over methods such as the Canny algorithm due to the coupling of speed and required accuracy for this problem. In order to facilitate the detection of the edges, we recolored the lung images to a $50 \%$ black and white scheme with 50\% softening. After recoloration and softening, we ran the images through the Sobel algorithm implemented in Python, which returned a list of edge pixels for each image as coordinate pairs. We first transformed these pairs into a new coordinate system with the center of the image as the origin then categorized the pairs into subsections corresponding to sectors defined by angles. We then calculated values for the stretch factor, kinetic energy per square meter $\left((1 / 2) \rho_{m} v^{2} H\right)$, and radial velocity in each radial direction.

Next, we applied nonlinear regression using the Levenberg-Marquadt algorithm on each subsection in order to extract the three material parameters $c_{1}, c_{2}$, and $c_{3}$ as well as the corresponding coefficients of determination $\left(R^{2}\right.$ values). We fit the velocity and kinetic energy data to the Denny-Schroter second-order Taylor series approximation. After we determined these parameters, they were classified as either stable or unstable corresponding to the previously derived stability criteria. Lastly, our program recolored the boundary of the lung parenchyma based on the stability of 


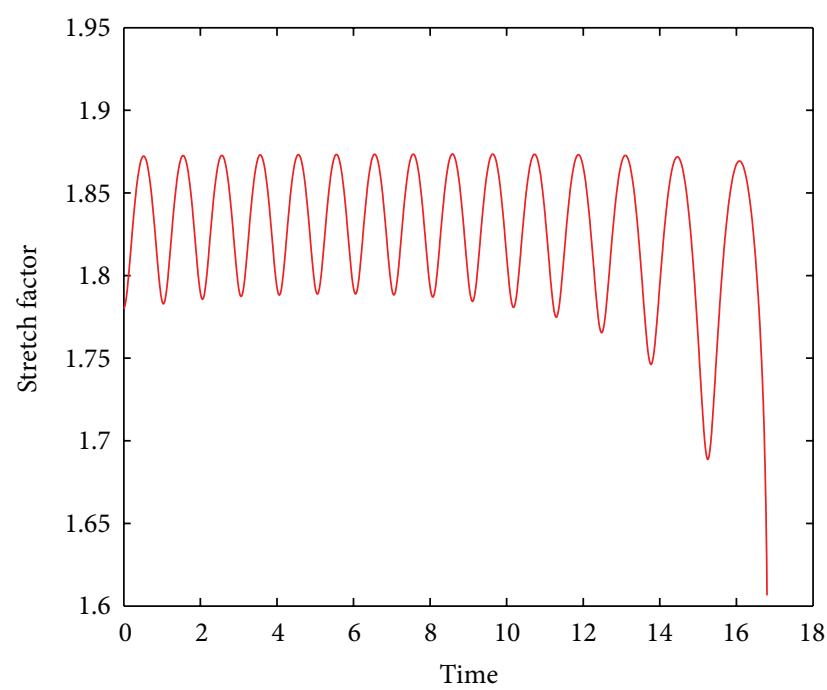

(a)

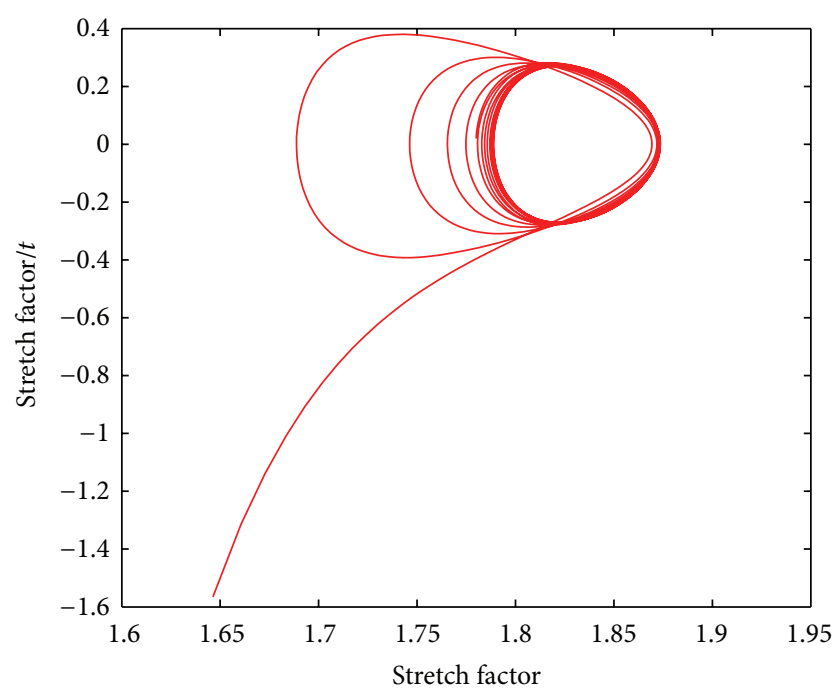

(b)

Figure 9: Collagen-elastin dynamics may lead to escapes from stable orbits. (a) The graph shows $\lambda(\tau)$ with the additional collagen-elastin dynamics calibrated with $A_{c}=100, A_{e}=50, k_{1}=1 \times 10^{7}, k_{2}=1 \times 10^{3}$, and $n=1$. The point of rupture occurs at the final peak of oscillation at $\tau \approx 16$. (b) The phase plane reflects the transition from stability to instability as the trajectory veers off from stable orbits.

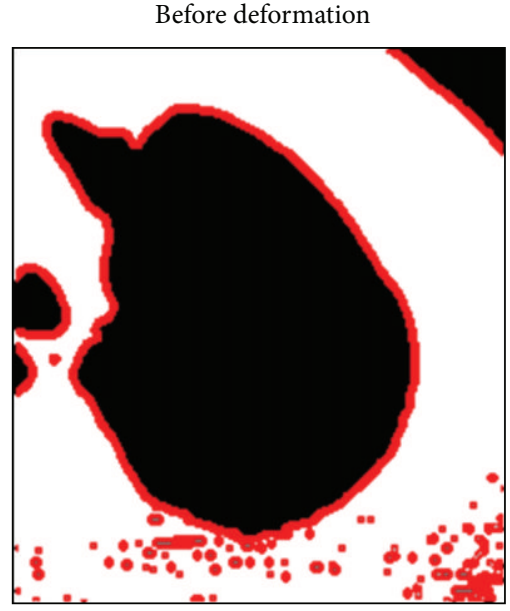

(a)

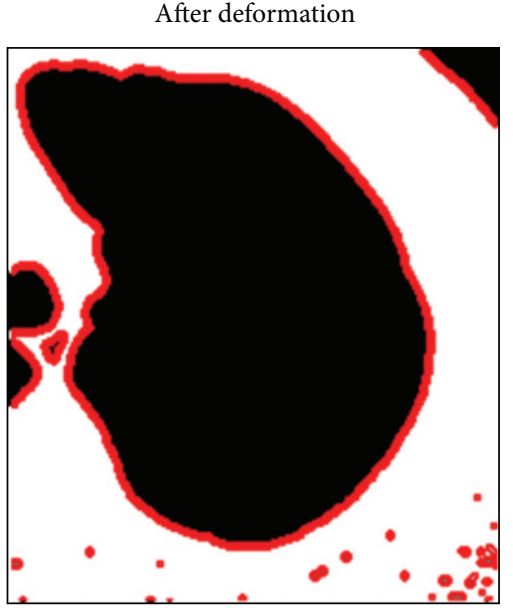

(b)

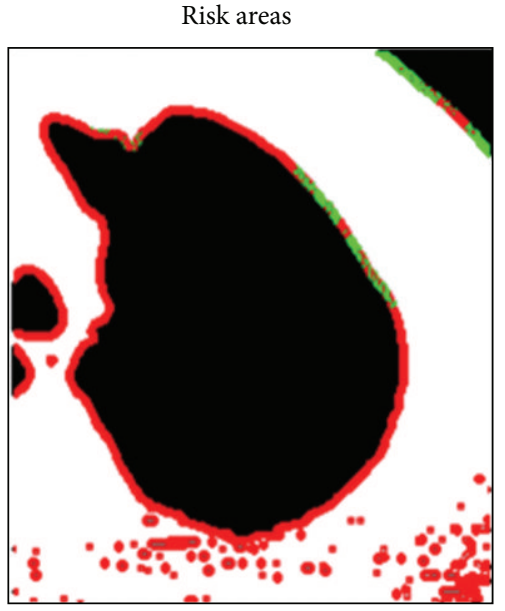

(c)

FIGURE 10: Edge detection and risk analysis. (a) and (b) show a lung before and after deformation, with red pixels indicating edge pixels detected by the Sobel Edge Detection algorithm. (c) shows the results of the nonlinear regression analysis performed on different sectors of the lung. Areas characterized as unstable, and thus at risk for lung collapse, are colored in red, whereas areas characterized as stable are colored in green.

the corresponding subsections, with green corresponding to stable subsections and red corresponding to unstable subsections. The general schema for our computational algorithm is shown in Figure 10. Thus, our algorithm is streamlined towards interpolating material parameters from deformations in lung CT scan data and identifying specific areas of risk in the lung parenchyma.

Motion artifacts, while important and are often observed in CT data acquisition, are not considered in this paper. The images are represented using intensity values at various pixel locations. This paper used only one dataset as a proof of concept. Our algorithm looks at small sections of the lung boundary, allowing for the isotropic assumption to be valid. However, it must be noted that this assumption must be removed for more general studies.

\section{Conclusion and Future Directions}

Our proposed biomechanical model for lung parenchymal lesions demonstrates that elastodynamic strain of the visceral pleural membrane from pulsatile air flow and changes in the constitutive protein composition of tissue are key pathological factors in pneumothorax. Through proving general 
instability results, our study demonstrated that these factors biject to physically unstable domains in the material parameter space for a general lesion. Specifically, our discovery that certain rate kinetics of collagen-elastin dynamics may lead to unstable mechanical configurations aligns well with clinical studies, which have found that certain connective tissue diseases lead to spontaneous pneumothorax [23, 24]. Future work on the theoretical biomechanics aspect of our model will incorporate COMSOL multiphysics simulation software integrated with finite element mesh processing to solve the problems of nonaxisymmetry and anisotropic lesion growth. Also, it would be interesting to study how variations in the dynamic viscosities affect the behavior of parenchymal lesions. Clinical studies have indicated that gravity is a significant factor on the air accumulated in the pleural cavity. We hope to consider these effects in future work. Quantification of material properties will require efficient inverse parameter estimation studies, which we hope to incorporate in the future. Such studies will help us to develop efficient simulation tools $[25,26]$. Other methods for lung deformation registration such as the dictionary learning approach used by Zhang et al. [27] will be considered.

Our algorithm for determining lesion stability from CT scan data relies on fitting our biomechanical model to deformations in a local tissue boundary. Using Sobel Edge Detection and CT scans from a lung image database, we could isolate unstable regions of local lung parenchymal tissue. The algorithm is a robust method for quantifying patient risk of pneumothorax and may be streamlined to work in the future with in vivo lung data collected from either ultrasound or CT scans. Overall, our computational solution for lung parenchymal lesion detection and patientspecific structural instability profiling is a feasible alternative diagnostic strategy and has the potential to surpass current diagnostic benchmarks for pneumothorax.

\section{References}

[1] P. B. Rich, G. R. Dulabon, C. D. Douillet et al., "Infrared thermography: a rapid, portable, and accurate technique to detect experimental pneumothorax," Journal of Surgical Research, vol. 120, no. 2, pp. 163-170, 2004.

[2] W. C. Yu, Y. C. Yeung, Y. Chang et al., "Use of endobronchial one-way valves reveals questions on etiology of spontaneous pneumothorax: report of three cases," Journal of Cardiothoracic Surgery, vol. 4, no. 1, article 1749, p. 63, 2009.

[3] G. P. Currie, R. Alluri, G. L. Christie, and J. S. Legge, "Pneumothorax: an update," Postgraduate Medical Journal, vol. 83, no. 981, pp. 461-465, 2007.

[4] C. A. Laituri, P. A. Valusek, D. C. Rivard et al., "The utility of computed tomography in the management of patients with spontaneous pneumothorax," Journal of Pediatric Surgery, vol. 46, no. 8, pp. 1523-1525, 2011.

[5] C. G. Ball, S. M. Hameed, D. Evans, J. B. Kortbeek, and A. W. Kirkpatrick, "Occult pneumothorax in the mechanically ventilated trauma patient," Canadian Journal of Surgery, vol. 46, no. 5, pp. 373-379, 2003.

[6] N. Nathan, J. Guilbert, M. Larroquet, M. Lenoir, A. Clement, and R. Epaud, "Efficacy of blebs detection for preventive surgery in children's idiopathic spontaneous pneumothorax," World Journal of Surgery, vol. 34, no. 1, pp. 185-189, 2010.

[7] R. C. Tai and G. C. Lee, "Isotropy and homogeneity of lung tissue deformation," Journal of Biomechanics, vol. 14, no. 4, pp. 243-252, 1981.

[8] E. Denny and R. C. Schroter, "A model of non-uniform lung parenchyma distortion," Journal of Biomechanics, vol. 39, no. 4, pp. 652-663, 2006.

[9] E. Denny and R. C. Schroter, "Relationships between alveolar size and fibre distribution in a mammalian lung alveolar duct model," ASME Journal of Biomechanical Engineering, vol. 119, no. 3, pp. 289-297, 1997.

[10] E. Denny and R. C. Schroter, "Viscoelastic behavior a lung alveolar duct model," ASME Journal of Biomechanical Engineering, vol. 122, no. 2, pp. 143-151, 2000.

[11] G. David and J. D. Humphrey, "Further evidence for the dynamic stability of intracranial saccular aneurysms," Journal of Biomechanics, vol. 36, no. 8, pp. 1143-1150, 2003.

[12] K. Jakuszkin, "Analysis of properties of a lung mechanical model during artificial ventilation using measurement station," Metrology and Measurement Systems, vol. 17, no. 3, pp. 427-438, 2010.

[13] A. Ben-Tal, "Simplified models for gas exchange in the human lungs," Journal of Theoretical Biology, vol. 238, no. 2, pp. 474495, 2006.

[14] A. D. Shah and J. D. Humphrey, "Finite strain elastodynamics of intracranial saccular aneurysms," Journal of Biomechanics, vol. 32, no. 6, pp. 593-599, 1999.

[15] K. Amjadi, G. G. Alvarez, E. Vanderhelst, B. Velkeniers, M. Lam, and M. Noppen, "The prevalence of blebs or bullae among young healthy adults: a thoracoscopic investigation," Chest, vol. 132, no. 4, pp. 1140-1145, 2007.

[16] O. Yetkin, I. Tek, F. Yetkin, and N. Numanoglu, "Role of pleural viscosity in the differential diagnosis of exudative pleural effusion," Respirology, vol. 12, no. 2, pp. 267-271, 2007.

[17] J. Rubins and H. Manning, "Pleural effusion workup," 2012, http://emedicine.medscape.com/article/299959-workup.

[18] S. Girnyk, A. Barannik, E. Barannik, V. Tovstiak, A. Marusenko, and V. Volokhov, "The estimation of elasticity and viscosity of soft tissues in vitro using the data of remote acoustic palpation," Ultrasound in Medicine and Biology, vol. 32, no. 2, pp. 211-219, 2006.

[19] K. Y. Volokh and D. A. Vorp, "A model of growth and rupture of abdominal aortic aneurysm," Journal of Biomechanics, vol. 41, no. 5, pp. 1015-1021, 2008.

[20] J. D. Humphrey and F. C. Yin, "A new constitutive formulation for characterizing the mechanical behavior of soft tissues," Biophysical Journal, vol. 52, no. 4, pp. 563-570, 1987.

[21] Y. Takubo, T. Hirai, S. Muro, K. Kogishi, M. Hosokawa, and M. Mishima, "Age-associated changes in elastin and collagen content and the proportion of types I and III collagen in the lungs of mice," Experimental Gerontology, vol. 34, no. 3, pp. 353364, 1999.

[22] D. S. Faffe and W. A. Zin, "Lung parenchymal mechanics in health and disease," Physiological Reviews, vol. 89, no. 3, pp. 759775, 2009.

[23] G. R. Junior, A. S. Rodrigues, and L. C. Meguins, "Thoracoscopic surgery for simultaneous bilateral spontaneous pneumothorax," The Internet Journal of Thoracic and Cardiovascular Surgery, vol. 12, no. 1, 2008. 
[24] H. J. Metivier, R. Masse, F. Vai, and R. G. Pariente, "Increased content of neutrosoluble collagen and dialyzable hydroxyproline in panacinar emphysema and spontaneous pneumothorax," Experimental Lung Research, vol. 3, no. 2, pp. 129-136, 1982.

[25] A. Santhanam, C. Fidopiastis, K. Langen, S. Meeks, P. Davenport, and J. P. Rolland, "Real-time visualization of subject specific lung dynamics," IEEE Computer-based Medical Systems, 2006.

[26] W. P. Segars, T. S. Lee, and B. M. W. Tsui, "Simulation of motion defects in the 4D NCAT cardiac model," Journal of Nuclear Medicine, vol. 44, p. 142P, 2003.

[27] S. Zhang, Y. Zhan, and D. N. Metaxas, "Deformable segmentation via sparse representation and dictionary learning," Medical Image Analysis, vol. 16, no. 7, pp. 1385-1396, 2012. 


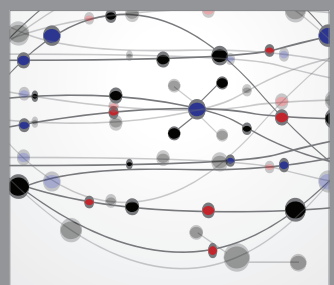

The Scientific World Journal
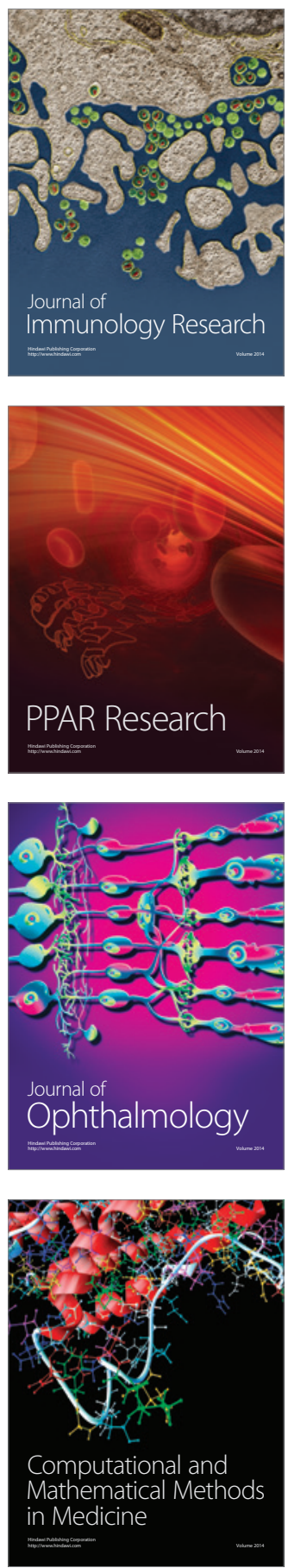

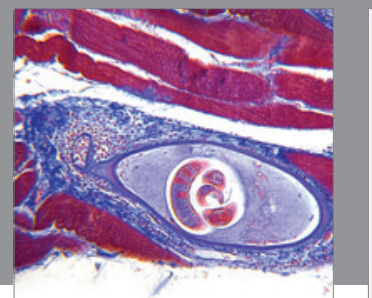

Gastroenterology

Research and Practice
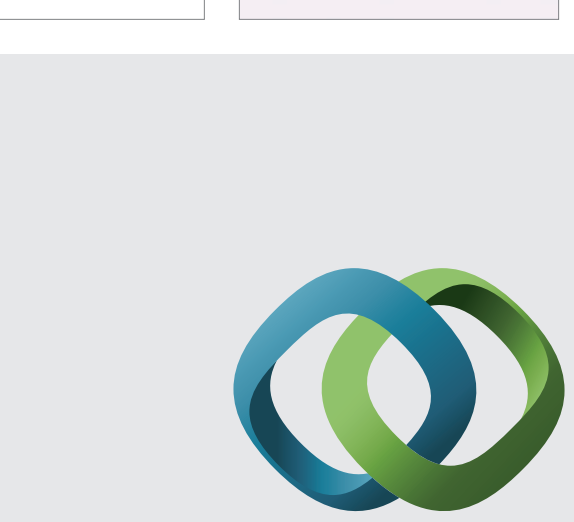

\section{Hindawi}

Submit your manuscripts at

http://www.hindawi.com
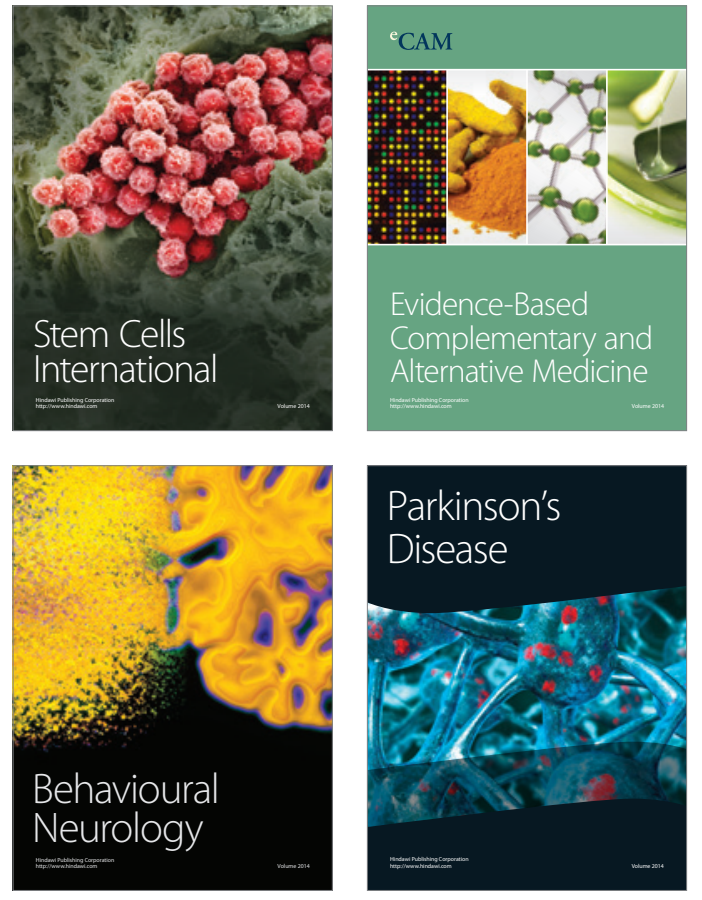
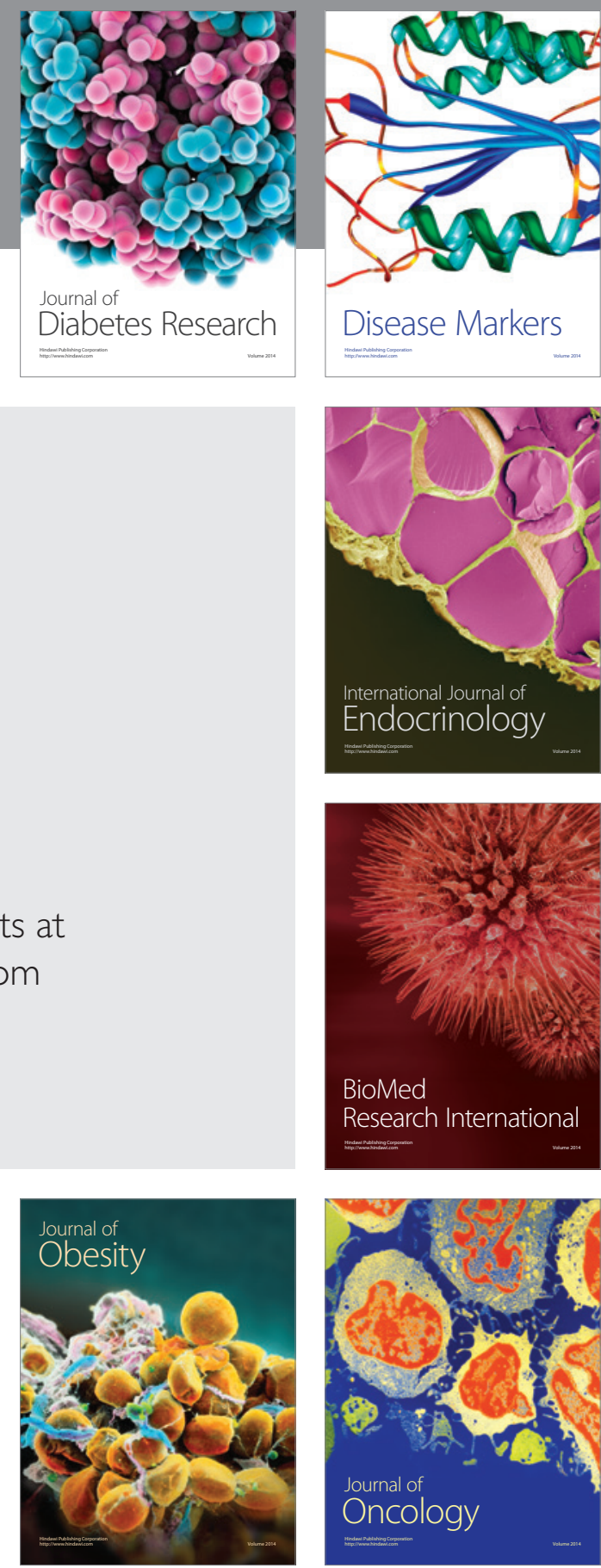

Disease Markers
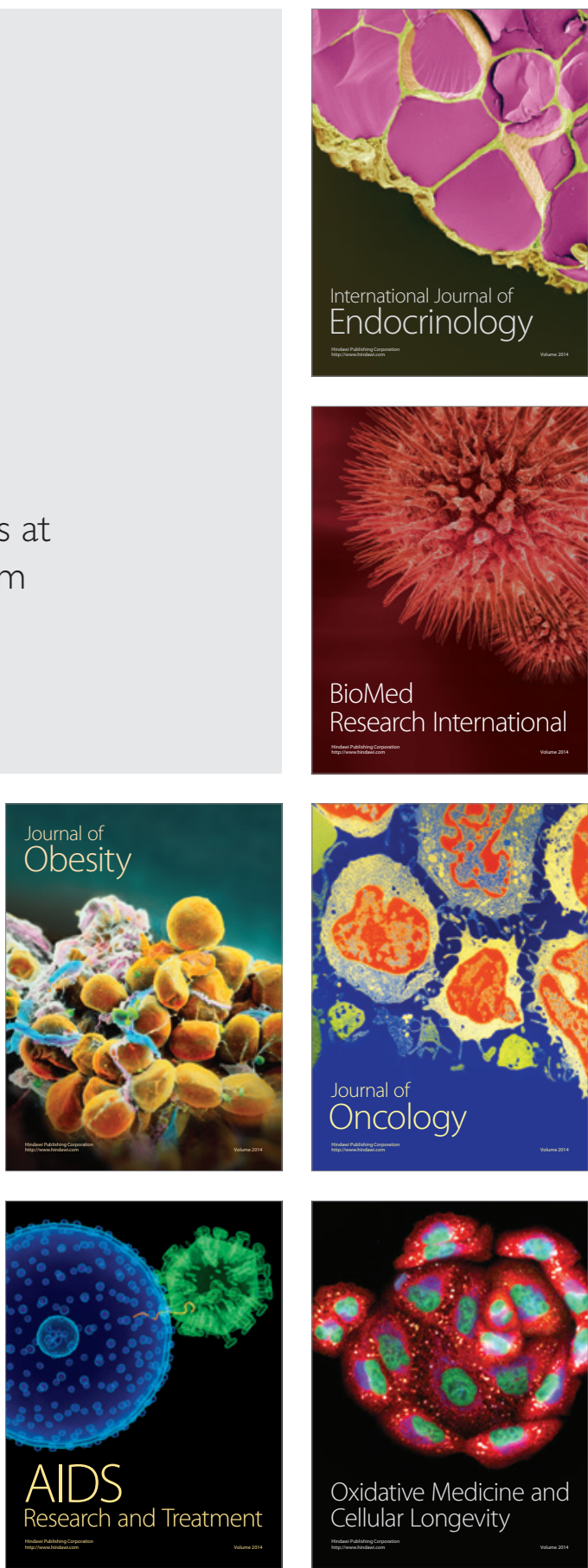